\title{
Cytotoxic Edema in Posterior Reversible Encephalopathy Syndrome: Correlation of MRI Features with Serum Albumin Levels
}

\author{
B. Gao, B.X. Yu, R.S. Li, G. Zhang, H.Z. Xie, F.L. Liu, and C. Lv
}

\begin{abstract}
BACKGROUND AND PURPOSE: Posterior reversible encephalopathy syndrome is a clinicoradiologic entity with typical MR imaging showing predominant vasogenic and occasional cytotoxic edema. It is unclear whether MR imaging correlates with levels of serum albumin. We determined potential risk factors for development of cytotoxic edema in posterior reversible encephalopathy syndrome.
\end{abstract}

MATERIALS AND METHODS: Seventy-nine cases with typical clinical symptoms and characteristic neuroradiologic findings conformed to posterior reversible encephalopathy syndrome diagnostic criteria and were included in this study. FLAIR, DWI, and ADC maps were interpreted to evaluate the severity and type of edema. MR imaging was correlated with the levels of serum albumin, and cytotoxic edema was compared with the location and severity of brain edema.

RESULTS: Pure vasogenic edema was found in 53 cases (67.09\%), and vasogenic edema complicated with cytotoxic components, in 26 patients (32.91\%). There was no difference in serum albumin levels between patients with cytotoxic components and those with vasogenic edema $(P=$.983). There was a significant difference in the edema scale scores between patients with cytotoxic edema and those with vasogenic edema $(P=.006)$. The percentage of cytotoxic edema located in the area with higher scale scores of edema was significantly larger than that in areas with lower scale scores of edema $(P=.002)$.

CONCLUSIONS: Serum albumin may contribute to the development of edema in PRES but is not a decisive factor for edema type. Cytotoxic edema in posterior reversible encephalopathy syndrome is probably related to regional decreased perfusion and arteriolopathy. Further work should be undertaken to discover the pathophysiologic mechanisms involved.

ABBREVIATIONS: COP = colloid osmotic pressure; PRES = posterior reversible encephalopathy syndrome

$\mathbf{P}$ osterior reversible encephalopathy syndrome (PRES) is a clinicoradiologic entity presenting with headache, visual blurring, alteration of mental state, and acute onset of seizures. MR imaging typically demonstrates evidence of vasogenic edema in the subcortical white matter and cortex, which predominantly involves the bilateral parieto-occipital lobes. ${ }^{1,2}$ DWI most com-

Received January 9, 2015; accepted after revision February 28.

From the Department of Radiology (B.G., B.X.Y., R.S.L., G.Z., H.Z.X., F.L.L.), Yantai Yuhuangding Hospital, Qingdao University, Shandong Province, China; Department of Radiology (B.G.), Zhongda Hospital, Southeast University, Nanjing, China; and Department of Neurology (C.L.), Yantai City Yantaishan Hospital and Yantai SinoFrance Friendship Hospital, Shandong Province, China.

B. Gao and B.X. Yu contributed equally to this article and are co-first authors. This work was supported by National Natural Science Foundation of China (Grant No. 81471645).

Please address correspondence to Cui Lv, MD, Department of Neurology, Yantai City Yantaishan Hospital and Yantai Sino-France Friendship Hospital, Yantai 264001, Shandong Province, China; e-mail: ytbrian@126.com

- Indicates open access to non-subscribers at www.ajnr.org

http://dx.doi.org/10.3174/ajnr.A4379 monly shows isointensities in the region of vasogenic edema as demonstrated by abnormalities on $\mathrm{T} 2$-weighted imaging or FLAIR images. However, although referred to as a reversible process, foci of reduced diffusion have been described in 17\%-26\% of patients with PRES. ${ }^{3,4}$ Cytotoxic edema in or outside the massive vasogenic edema was found in a relatively lower proportion of patients with PRES and could be differentiated from vasogenic edema by using DWI. Early detection of cytotoxic edema in PRES is critical to prevent vasogenic edema from progressing into irreversible insult.

Several theories exist as to why regions of reduced diffusion are seen in some cases of PRES. ${ }^{5}$ The most popular theory is hyperperfusion causing severe mass effect from vasogenic edema with compression of local microcirculation..$^{6,7}$ Ay et $\mathrm{al}^{6}$ suggested that increased tissue pressure eventually impairs the microcirculation and leads to ischemia in areas of massive vasogenic edema. Covarrubias et $\mathrm{al}^{7}$ found that cytotoxic edema developed in the cortex immediately adjacent to the area with intensely elevated ADC values in subcortical white matter, which is consistent with a 
heavy burden of fluid in the interstitium. It has also been suggested that edema with resultant vasoconstriction or vasospasm (with or without subarachnoid hemorrhage) leads to cytotoxicity. ${ }^{8,9}$ Bartynski et $\mathrm{al}^{9}$ confirmed a high incidence of vascular abnormality in PRES (focal vasoconstriction, vasodilation, and string-of-beads appearance) at conventional angiography and demonstrated these observations and the reversibility at MRA. It remains unclear why vasogenic edema in PRES develops into cytotoxic edema. Unchecked vasogenic edema may develop into cytotoxic edema or irreversible insults. Pirker et $\mathrm{al}^{10}$ recently reported that vasogenic edema in PRES was much more frequent in patients with decreased levels of serum albumin, whereas cytotoxic components occurred more frequently in patients with normal levels. However, the extent to which vasogenic edema is associated with reduced serum albumin levels remains unclear, as does the cause of cytotoxic edema development.

Some studies have demonstrated that cytotoxic edema is more commonly seen in PRES. In the present study, we determined the association of edema type with the levels of serum albumin in patients with PRES. We hypothesized the following: 1) the level of serum albumin would correlate with edema type, 2) severe vasogenic edema could progress into cytotoxic edema, and 3) the location of cytotoxic edema would relate to the extent and location of vasogenic edema.

\section{MATERIALS AND METHODS}

Seventy-nine patients clinically diagnosed with PRES were identified during admission to Yantai Yuhuangding Hospital from January 1, 2008, to October 31, 2014, and were pooled with a data base containing complete electronic clinical records. Our institutional review board approved this study with a waiver of informed consent. The definition of PRES used in this study fulfills the following criteria: 1) presentation with acute clinical symptoms such as headache, altered mental status, seizures, or visual disturbance with or without elevated systemic blood pressure; 2) presence of known risk factors such as hypertension, eclampsia, renal failure, use of immunosuppressive drugs, or other known causes; 3) distributions of T2WI or T2-FLAIR hyperintensities compatible with typical PRES imaging patterns; 4) clinical and imaging abnormalities mostly or completely resolved after proper therapy; and 5) other possible causes of encephalopathy or vasogenic edema ruled out. Exclusion criteria included the inability to complete the imaging examination or severe motion degradation resulting in nondiagnostic images and known systematic factors that would affect the results of blood sampling.

The presence or absence of hypertension and evidence of endothelial injury and hemolysis (lactate dehydrogenase, liver function, kidney function) was recorded. Serial venous blood samples were collected at admission to our hospital at symptom onset. The time interval from symptom onset to the collection of blood samples was recorded in all patients. MR imaging was performed at our institution on 3T MR imaging equipment. Conventional axial T1WI, T2WI, FLAIR, and DWI sequences were acquired, and coronal or sagittal T2WI scanning was available for all patients. MR imaging was performed as soon as possible on the day of symptom onset, and the time interval from symptom onset to MR imaging examinations was recorded in all patients.
All MR imaging results were reviewed by 2 senior neuroradiologists (G.B., L.F.-l.) blinded to clinical data. Each reviewer thoroughly interpreted the location, distribution, and severity of the signal abnormality; the presence of hemorrhage; and reduced diffusion. The locations of hyperintensities were defined as frontal lobe, parieto-occipital region, temporal lobe, basal ganglia, cerebellum, brain stem, deep white matter, cortical zone, subcortical zone, or watershed zone. We scored 1 for any region with signal abnormality in the bilateral hemispheres. The sum of scores of all affected locations in an individual patient provided the score of brain edema. The severity of the abnormality was graded on a scale of $0-3$ based on FLAIR images ( 0 , normally appearing brain parenchyma; 1 , subtle signal abnormalities that were only faintly visible; 2, larger confluent areas of hyperintense abnormalities that were easily perceptible; and 3, complete involvement of the brain region) modified from McKinney et al. ${ }^{3}$ Images of patients with multiple MR examinations were analyzed for resolution of imaging abnormalities.

The type of edema can be categorized as vasogenic or cytotoxic. Vasogenic edema was determined by T2WI hyperintensity without reduced diffusion, while cytotoxic edema was considered in cases of T2WI hyperintensity with reduced diffusion. When we measured molecular motion with DWI, only the ADC value could be calculated. Reduced diffusion was determined by the ADC value, which was calculated as follows: $\mathrm{ADC}=\ln \left(S_{0} / S_{1}\right) /\left(b_{1}-\right.$ $b_{0}$ ), where $S_{0}$ and $S_{1}$ are the image intensities at b-values of $b_{0}$ and $b_{1}$ respectively.

The PASW statistical package (IBM, Armonk, New York) was used for all statistical analyses. Data normality was tested with the Shapiro-Wilk test. Normally distributed data are expressed as mean $\pm \mathrm{SD}$, and data that are not normally distributed are presented as median (interquartile range). Intergroup comparison was performed by using the Student $t$ test for normally distributed variables and the Mann-Whitney $U$ test for variables not normally distributed.

\section{RESULTS}

Seventy-nine patients (34 male, 45 female; age range, 7-66 years; mean age, $32.1 \pm 11.5$ years) with complete in-hospital clinical records were included in this study. Thirty-five (44.3\%) of all 76 cases were patients with eclampsia or pre-eclampsia, 25 cases $(31.6 \%)$ were patients with chronic renal failure, 14 (17.7\%) were patients with hypertension, and $5(6.3 \%)$ had different clinical conditions (autoimmune disease in 3 and cancer chemotherapy in 2). Clinical symptoms included headache in 68 patients $(86.07 \%)$, altered mental state in $59(74.68 \%)$, seizures in 40 (50.63\%), visual disturbances in 25 (31.64\%), and focal neurologic deficit in 11 patients (13.92\%).

All patients had typical clinical symptoms and characteristic MR imaging findings of PRES. The parietal and occipital regions were involved in all 79 (100\%) patients; the frontal region, in 70 (88.61\%); and the temporal region, in 36 (45.57\%). The cerebellum was involved in $30(37.97 \%)$ patients; the deep gray matter, in $22(27.85 \%)$; the brain stem, in $15(18.99 \%)$; the periventricular region, in 12 (15.19\%); and the corpus callosum, in 10 (12.66\%) patients. Hemorrhages were present in $16(20.25 \%)$ patients. Nine had intraparenchymal hemorrhages, 5 had cerebral microb- 

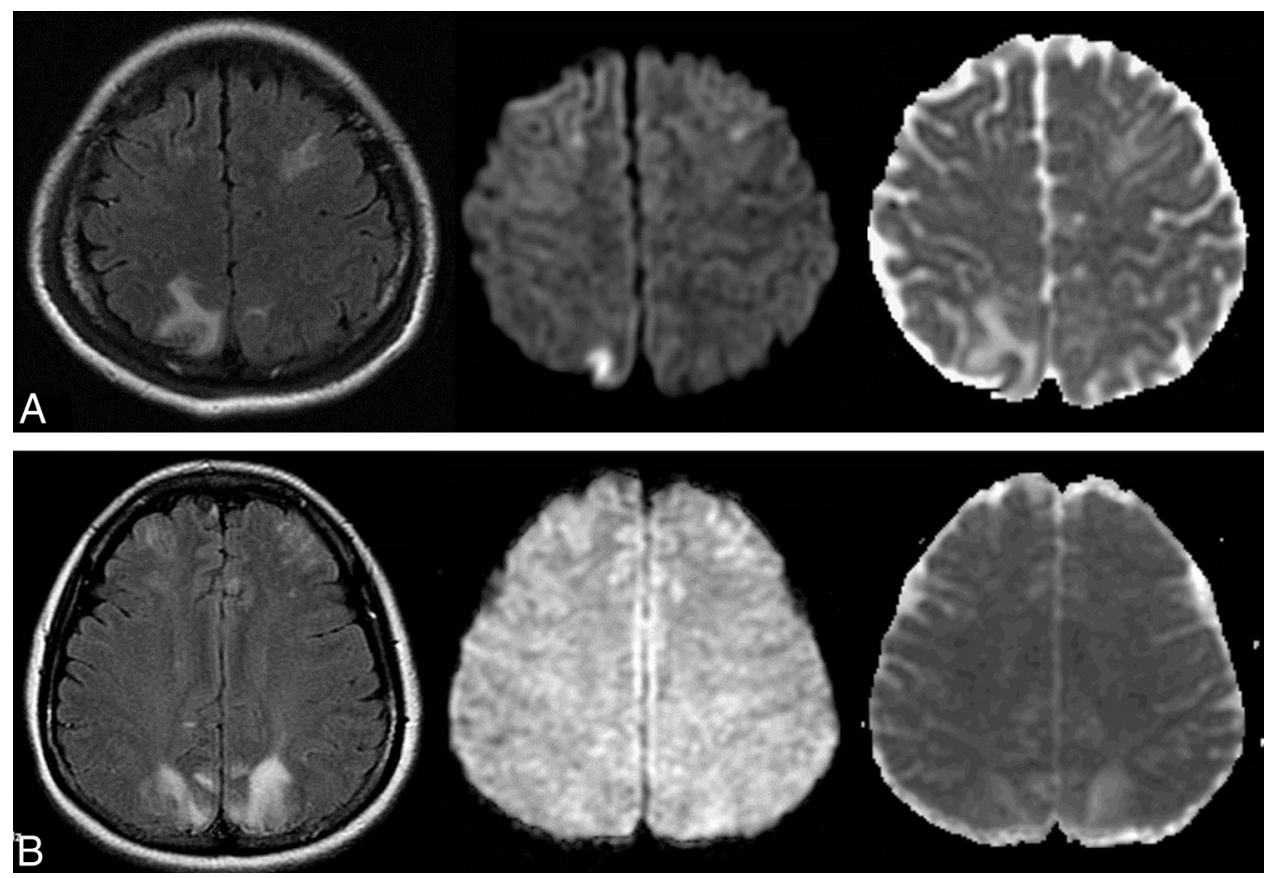

FIG 1. Comparison of serum albumin levels between patients with PRES with cytotoxic edema and those only with vasogenic edema. A, A 26-year-old pregnant woman presented with eclampsia, with a serum albumin level of $37 \mathrm{mg} / \mathrm{dL}$. Hyperintense abnormalities are found in bilateral frontoparietal lobes on FLAIR (left), hyperintense on DWI (middle) and hypointense on ADC (right), and are also noted in the right parietal lobe, indicating cytotoxic edema. B, A 22-year-old woman with systemic vasculitis with a serum albumin level of $34.2 \mathrm{mg} / \mathrm{dL}$ and blood pressure of $175 / 110 \mathrm{~mm} \mathrm{Hg}$ at onset. Symmetric hyperintensities are found in the bilateral frontoparietal lobes on FLAIR (left), isointense on DWI (middle) and hyperintense on ADC (right), indicating vasogenic edema.

leeds, and 2 had subarachnoid hemorrhages. MR venography examinations were performed in 56 patients $(70.89 \%)$ and did not reveal an abnormality in any patient.

After analyzing all the study images including T1WI, T2WI, FLAIR, DWI sequences, and ADC maps, we found pure vasogenic edema in 53 cases $(67.09 \%)$ and vasogenic edema complicated with cytotoxic components in 26 patients (32.91\%). The type of edema was not associated with the interval between the onset of symptoms and MR imaging examinations $(P=.58$, Mann-Whitney $U$ test) or with the interval between the onset of symptoms and blood sample collection ( $P=.34$, Mann-Whitney $U$ test). There was no difference in the time from presentation to imaging or to blood sample collection across subjects $(P=.61$, MannWhitney $U$ test).

Serum albumin levels were normally distributed in all patients with PRES ( $P=.059$, Shapiro-Wilk test), and a decrease of serum albumin concentration $(<35 \mathrm{mg} / \mathrm{mL})$ was observed in $67 / 79$ patients $(84.8 \%$; mean, $23.6 \pm 5.8 \mathrm{mg} / \mathrm{dL})$. There was no difference in the serum albumin levels between patients with cytotoxic edema and those without ( $P=.983$, Mann-Whitney $U$ test) (Fig 1). There was no significant difference in the percentage of serum albumin decrease between patients presenting with cytotoxic edema $(21 / 26,80.77 \%)$ and those without $(46 / 53,86.79 \%)$ (Table). The scale scores of edema in all patients were not normally distributed ( $P=.00$, Shapiro-Wilk test). The levels of serum albumin were not correlated with the scale scores of edema $(P=.267)$. There was no statistically significant difference in serum albumin levels between the eclamptic group and the chronic renal failure group $(P=.23)$, as well as the eclamptic
Comparison of clinical parameters in patients with present and absent cytotoxic edema

\begin{tabular}{|c|c|c|c|}
\hline Parameters & $\begin{array}{c}\text { Cytotoxic } \\
\text { Edema Present } \\
(n=26)\end{array}$ & $\begin{array}{l}\text { Cytotoxic } \\
\text { Edema Absent } \\
(n=53)\end{array}$ & $P$ Value \\
\hline Age $(y)$ & $34.51 \pm 11.42$ & $33.21 \pm 15.01$ & .74 \\
\hline Sex & $11 / 15$ & $21 / 32$ & .077 \\
\hline Cause $^{a}$ & $4 / 33$ & $5 / 46$ & .19 \\
\hline MAP (mm Hg) & $138.51 \pm 28.63$ & $124.83 \pm 30.22$ & .13 \\
\hline ALB (mg/mL) & $522.00 \pm 241.47$ & $388.00 \pm 191.18$ & .98 \\
\hline ALB decreased ratio & $22 / 26$ & $45 / 53$ & .12 \\
\hline SBE & $20.36 \pm 11.43$ & $10.24 \pm 7.20$ & $.003^{b}$ \\
\hline
\end{tabular}

Note:-MAP indicates mean arterial pressure; ALB, albumin; SBE, score of brain edema.

a Percentage of patients with eclampsia or pre-eclampsia.

b Significant.

group and the hypertensive group $(P=.45)$, and the chronic renal failure group and hypertensive group $(P=.17)$.

Scores of brain edema in all patients were not normally distributed ( $P=.037$, Shapiro-Wilk test), and serum albumin levels were not correlated with scores of brain edema $(P=$ .145). There was a significant difference in scores of brain edema between patients with cytotoxic components and those without $(Z=2.969, P=.003$, Mann-Whitney $U$ test). The extent of edema in patients with cytotoxic components was significantly larger than that of patients without (Table). The percentage of cytotoxic edema located in the area with higher scale scores of edema $(=3)$ was significantly larger than that in those with lower scale scores $(\leq 2)(P=.002$, Fisher exact test $)$ (Fig 2). 

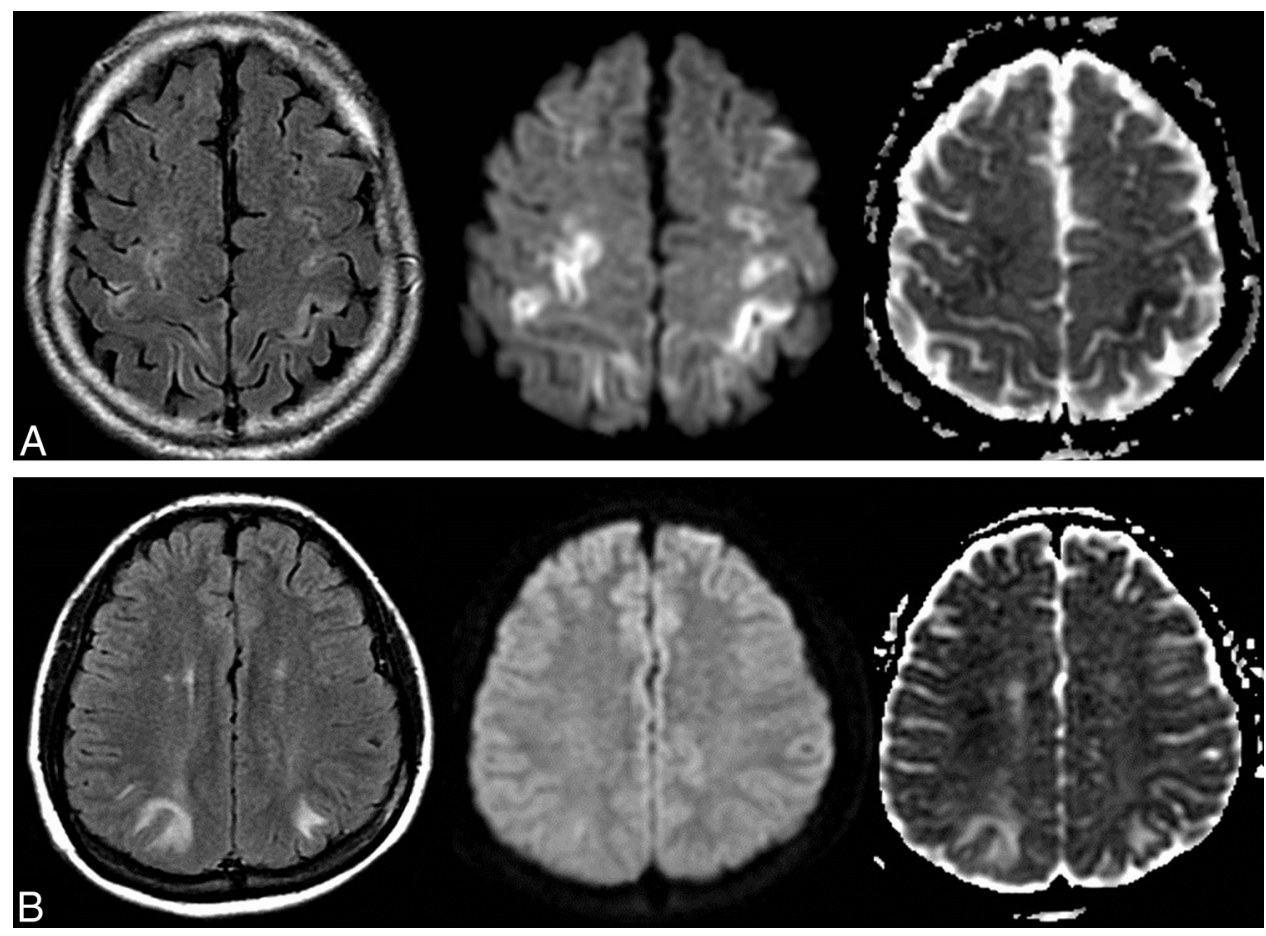

FIG 2. Comparison of the scores of brain edema between patients with PRES with cytotoxic edema and those only with vasogenic edema. $A$, A 25-year-old woman with eclampsia and seizures after cesarean delivery. FLAIR image shows symmetric confluent heterogeneous hyperintensities in the bilateral, frontal, and parietal lobes (left) with a scale score 3 of edema severity and marked hyperintensity in the adjacent cortex on DWI (middle), with hypointensity on the ADC map (right), indicating cytotoxic edema. B, A 27-year-old man with hypertension and nephritis had headache with blood pressure of 180/120 mm. Symmetric hyperintensities are found in the bilateral occipital lobes on FLAIR (left) with a scale score 1 of edema severity, isointense on DWI (middle) and hyperintense on ADC (right), indicating vasogenic edema.

\section{DISCUSSION}

In the present study, decreased levels of serum albumin were found in $84.8 \%$ of all patients, suggesting that a change in serum albumin levels may be one of the potential factors resulting in edema development in PRES. Serum albumin is a main contributor to colloid osmotic pressure (COP). Decrease of serum albumin levels would result in retention of intravascular fluid by reducing perfusion pressure. ${ }^{11}$ In conditions with endothelial damage due to inflammatory processes, reduction of COP may facilitate fluid extravasation and edema development. ${ }^{10}$ Vasogenic edema can be aggravated by a marked decrease of COP, as shown by Pirker et al. ${ }^{10}$ However, we observed that serum albumin concentration was not associated with edema type and was decreased in patients with cytotoxic edema. Albumin contributes to up to $80 \%$ of COP in healthy subjects. However, its contribution toward COP is only $17 \%$ in critically ill individuals. ${ }^{12}$ Reduction of COP alone would not cause edema in the normal brain tissue, which is largely due to the properties of capillary endothelium that constitute the BBB. ${ }^{13}$ The failure of autoregulatory mechanisms involved in the maintenance of $\mathrm{CBF}$ might also lead to hyperperfusion and vasogenic edema in the presence of excessive elevations in systemic blood pressure. Low serum albumin may be a surrogate marker of disease severity rather than an indicator of low COP. Therefore, when one treats patients with PRES with hypoalbuminemia, the underlying cause of hypoalbuminemia should probably be treated first. Two of our patients hardly improved with hypoalbuminemia treatment. While improving hypoalbuminemia may or may not help the patient, correction of the underlying disorder is more critical than merely reversal of hypoalbuminemia. ${ }^{14}$

The pathogenesis of brain lesions in PRES is not fully understood, and 2 opposing theories have been proposed. ${ }^{15}$ Endothelial dysfunction seems to better account for the mechanisms of PRES, given that both vasogenic and cytotoxic edema could occur in PRES, as well as hypoperfusion or hyperperfusion. ${ }^{16}$ Forced dilation of cerebral arteries and disruption of the integrity of the BBB are considered the most important mechanisms underlying vasogenic edema observed in patients with PRES and hypertension. Alternatively, ischemia triggered by systemic inflammation, endothelial injury, and vascular dysfunction is also suggested to contribute to vasogenic edema observed in PRES, as well as active leakage of fluid due to other processes affecting the vascular wall. Parenchymal contrast enhancement, typically seen only with injury to the BBB, also supports this theory. Although most patients do not show any abnormal enhancement on postcontrast T1WI, it has been reported to occur in $21 \%-38 \%$ of patients with PRES. ${ }^{3,4}$ Typically, the enhancement is a mild, gyri form, and there is leptomeningeal or cortical enhancement within the lesions on postcontrast imaging studies. More commonly than in adults, contrast enhancement in children showed that pathophysiology was related to the BBB breakdown and also supported the theory of toxic endothelial injury. ${ }^{17} \mathrm{BBB}$ dysfunction might not only be restricted to areas with apparent lesions but could also involve the normal-appearing cerebral tissue, and even the retinal circulation. ${ }^{18}$ Therefore, endothelial injury or injury to the $\mathrm{BBB}$ is

AJNR Am J Neuroradiol 36:1884-89 Oct 2015 www.ajnr.org 
probably more likely than the other mechanism to account for the spectrum and variety of physiologic findings noted in PRES.

In this study, cytotoxic components in vasogenic edema were found in $33 \%$ of all patients, a slightly higher percentage compared with $17 \%-26 \%$ of patients with PRES with foci of reduced diffusion in prior studies of these patients. ${ }^{3,4}$ This slightly higher percentage may be attributable to either case selection criteria or case source.

Several studies have reported radiologic findings of cytotoxic edema or ischemia in PRES, ${ }^{3,4}$ which were associated with fibrinoid necrosis and microinfarction in pathologic studies. ${ }^{19,20}$ Cytotoxic edema manifests as DWI hyperintensity with decreased $\mathrm{ADC}$ values. Moreover, in patients with cytotoxic edema, there is partial reversal or nearly complete resolution of the abnormality, and even patients with cytotoxic edema would not show conversion to infarction or may have mild atrophy. Cytotoxic edema in the acute phase does by no means imply conversion to infarction, and DWI does not seem to be a valid prognostic marker. ${ }^{21}$ The value of DWI and ADC maps in predicting the clinical prognosis of PRES warrants further investigation. ${ }^{22}$ Cytotoxic edema in PRES may be attributable to cerebral vasospasm, with endothelial activation or injury leading to reduced CBF with consequent hypoxia, as described in several PRES studies using MR imaging or conventional angiography. ${ }^{9}$ Most interesting, a recent study reported that the high incidence of cerebral microbleeds was not correlated with edema severity/extent and the presence of DWIpositive findings. ${ }^{23}$ Atypical neuroimaging appearances, such as intracranial hemorrhage, cytotoxic edema, and abnormal enhancement, may reflect endothelial injury or disruption and would contribute to early diagnosis and prognosis evaluation. ${ }^{24}$ Vascular imaging and perfusion imaging would be helpful to further elucidate the mechanism of edema development in PRES. ${ }^{25}$

We showed that the scale scores of edema in patients with cytotoxic components were significantly higher than those in patients with pure vasogenic edema, and the percentage of patients with cytotoxic edema in areas with higher scale scores was significantly higher than that in those areas with lower scale scores. The presence of ischemia within areas of vasogenic edema has been observed in several retrospective studies. ${ }^{3,4,6,7}$ However, to the best of our knowledge, the development of ischemia with an increasing degree of brain edema has not been well-described. A correlation between the presence of vasculopathy and ischemia was recently reported, suggesting a causal relationship between cytotoxic edema and larger areas of vasogenic edema. ${ }^{26}$ The normally protective vasoconstrictive response can progress into vasospasm, leading to local hypoxia, BBB disruption, fluid extravasation, and subsequent edema. ${ }^{27}$ Enhanced systemic endothelial activation (swelling) and leukocyte trafficking and vasoconstriction, alone or in combination, may result in brain and systemic hypoperfusion. ${ }^{15}$ Therefore, higher scale scores of brain edema often develop and progress into ischemia. We also found that most cytotoxic edema was superimposed on, or adjacent to, the larger areas of vasogenic edema. A larger area of edema is typically contiguous and crosses large vessel boundaries, rather than being a watershed pattern. ${ }^{26}$ The mass effect induced by larger areas of vasogenic edema would compress or shift the vessels passing through or around the edema region, which may result in regional hypoperfusion, elevation of tissue pressure, and reduction of CBF to ischemic levels, as well as vasoconstriction. ${ }^{5,28}$ Subsequently, the areas surrounding marked vasogenic edema may progress to cytotoxic edema. We agree with the proposal that cytotoxic edema in PRES is probably related to local decreased perfusion and arteriolopathy. ${ }^{28}$ However, further work should be performed to uncover the pathophysiologic mechanisms involved.

Eclampsia or pre-eclampsia is the primary clinical cause of PRES. During a normal pregnancy, the BBB adapts to prevent circulating permeable factors from entering the brain. Plasma from women with pre-eclampsia has been found to increase the permeability of the BBB; the latter would permit the passage of damaging antivasogenic and antiendothelial proteins into the brain and result in neurologic symptoms of eclampsia. ${ }^{29,30} \mathrm{~A}$ higher percentage $(44.3 \%)$ of patients with eclampsia was also found in this study than was typically seen in past studies, which may be related to either our university hospital type or case-selection criteria. Ishikura et $\mathrm{al}^{31}$ even reported 7 pediatric patients with nephrotic syndrome who developed PRES and had low levels of serum albumin, implying a possible correlation between them. In these patients, PRES rapidly abated after the addition of albumin, despite further treatment with cytotoxic substances. Infusion of human serum albumin at early and reversible phases to increase oncotic pressure and restore antioxidative potential could theoretically prevent widespread oxidative and/or ischemic damage to vulnerable tissue in PRES. ${ }^{10}$ Many cases of acute toxic leukoencephalopathy may share common causes and pathophysiologic features with PRES. Reduced diffusion is typically seen in association with acute toxic leukoencephalopathy but less commonly in PRES, which could account for the differences of the mechanism and location in white matter involvement between the 2 processes. $^{32}$

Because clinical factors, imaging findings, and CSF laboratory results may influence the prognosis of PRES, a few limitations of this prospective study should be noted. First, selection bias toward the etiology of PRES may result in significant heterogeneity as to the relative proportion of patients for the study, and it is difficult to determine a single underlying etiology for PRES, often having multiple etiologies. Second, although vasculopathy is thought to play an important part in the pathophysiology of PRES, MR angiography was not routinely performed in our study. Additionally, the extent to which inflammatory changes contribute to vasculopathy has not been clarified. Third, because urine testing is not routinely performed at the onset in our patients with PRES, urine albumin concentrations could not be appreciated with serum albumin levels and neither could albumin concentration in CSF based on lumbar puncture. ${ }^{33}$ Prospective exploration is warranted to establish the association of biochemical values with unfavorable outcome in patients with PRES, and this might prove useful to clinicians of various disciplines involved in the care of these patients. ${ }^{34,35}$

\section{CONCLUSIONS}

Serum albumin may be a potential contributor to the development of edema in PRES, but it is not a decisive factor for edema type. Cytotoxic edema or ischemia is often seen in larger areas or to a higher extent of vasogenic edema, which is probably related to 
local decreased perfusion and arteriolopathy. Further work should be performed to uncover and understand the pathophysiologic mechanisms involved.

\section{ACKNOWLEDGMENTS}

The authors are grateful for the assistance of Dr Ri-ming Liu in the laboratory tests.

Disclosures: Bo Gao-RELATED: Grant: National Natural Science Foundation of China (Grant No. 81471645).

\section{REFERENCES}

1. Hinchey J, Chaves C, Appignani B, et al. A reversible posterior leukoencephalopathy syndrome. N Engl J Med 1996;334:494-500

2. Bartynski WS. Posterior reversible encephalopathy syndrome, Part 1: fundamental imaging and clinical features. AJNR Am J Neuroradiol 2008;29:1036-42

3. McKinney AM, Short J, Truwit CL, et al. Posterior reversible encephalopathy syndrome: incidence of atypical regions of involvement and imaging findings. AJR Am J Roentgenol 2007;189:904-12

4. Fugate JE, Claassen DO, Cloft HJ, et al. Posterior reversible encephalopathy syndrome: associated clinical and radiologic findings. Mayo Clin Proc 2010;85:427-32

5. Rykken JB, McKinney AM. Posterior reversible encephalopathy syndrome. Semin Ultrasound CT MR 2014;35:118-35

6. Ay H, Buonanno FS, Schaefer PW, et al. Posterior leukoencephalopathy without severe hypertension: utility of diffusion-weighted MRI. Neurology 1998;51:1369-76

7. Covarrubias DJ, Luetmer PH, Campeau NG. Posterior reversible encephalopathy syndrome: prognostic utility of quantitative diffusion-weighted MR images. AJNR Am J Neuroradiol 2002;23:1038-48

8. Henderson RD, Rajah T, Nicol AJ, Read SJ. Posterior leukoencephalopathy following intrathecal chemotherapy with MRA-documented vasospasm. Neurology 2003;60:326-28

9. Bartynski WS, Boardman JF. Catheter angiography, MR angiography, and MR perfusion in posterior reversible encephalopathy syndrome. AJNR Am J Neuroradiol 2008;29:447-55

10. Pirker A, Kramer L, Voller B, et al. Type of edema in posterior reversible encephalopathy syndrome depends on serum albumin levels: an MR imaging study in 28 patients. AJNR Am J Neuroradiol 2011;32:527-31

11. Quinlan G, Martin GS, Evans TW. Albumin: biochemical properties and therapeutic potential. Hepatology 2005;41:1211-19

12. Blunt MC, Nicholson JP, Park GR. Serum albumin and colloid osmotic pressure in survivors and nonsurvivors of prolonged critical illness. Anaesthesia 1998;53:755-61

13. Drummond JC. Colloid osmotic pressure and the formation of posttraumatic cerebral edema. Anesthesiology 2010;112:1079-81

14. Pulimood TB, Park GR. Debate: albumin administration should be avoided in the critically ill. Crit Care 2000;4:151-55

15. Bartynski WS. Posterior reversible encephalopathy syndrome, Part 2: controversies surrounding pathophysiology of vasogenic edema. AJNR Am J Neuroradiol 2008;29:1043-49

16. Marra A, Vargas M, Striano P, et al. Posterior reversible encephalopathy syndrome: the endothelial hypotheses. Med Hypotheses 2014; 82:619-22

17. Donmez FY, Guleryuz P, Agildere M. MRI findings in childhood PRES: what is different than the adults? Clin Neuroradiol 2014 Oct 8. [Epub ahead of print]
18. Ozkan E, Gocmen R, Topcuoglu MA, et al. Blood-retina-barrier disruption accompanying blood-brain-barrier dysfunction in posterior reversible encephalopathy syndrome. J Neurol Sci 2014; 346:315-17

19. Decker DA, Falchook AD, Yachnis AT, et al. Radiographic and pathologic findings in an atypical brainstem variant of reversible posterior leukoencephalopathy syndrome. Neurologist 2009;15: $364-66$

20. Jacquot $C$, Glastonbury CM, Tihan T. Is posterior reversible encephalopathy syndrome really reversible? Autopsy findings 4.5 years after radiographic resolution. Clin Neuropathol 2015;34:26-33

21. Kastrup O, Schlamann M, Moenninghoff C, et al. Posterior reversible encephalopathy syndrome: the spectrum of MR imaging patterns. Clin Neuroradiol 2015;25:161-71

22. Gao B, Lv C. Posterior reversible encephalopathy syndrome in 46 of 47 patients with eclampsia: beyond it. Am J Obstet Gynecol 2014;211:83-84

23. McKinney AM, Sarakiya B, Gustafson C, et al. Detection of microhemorrhage in posterior reversible encephalopathy syndrome using susceptibility-weighted imaging. AJNR Am J Neuroradiol 2012; 33:896-903

24. Lv C, Gao B. Can clinical and MRI findings predict the prognosis of variant and classical type of posterior reversible encephalopathy syndrome (PRES)? Still a challenge. Acta Radiol 2015;56:NP3-4

25. Hinchey JA. Reversible posterior leukoencephalopathy syndrome: what have we learned in the last $\mathbf{1 0}$ years? Arch Neurol 2008; 65:175-76

26. Li Y, Gor D, Walicki D, et al. Spectrum and potential pathogenesis of reversible posterior leukoencephalopathy syndrome. J Stroke Cerebrovasc Dis 2012;21:873-82

27. Williams KP, Wilson S. Persistence of cerebral hemodynamic changes in patients with eclampsia: a report of three cases. Am J Obstet Gynecol 1999;181:1162-65

28. Tamaki K, Sadoshima S, Baumbach GL, et al. Evidence that disruption of the blood-brain barrier precedes reduction in cerebral blood flow in hypertensive encephalopathy. Hypertension 1984;6(2 pt 2):I75-81

29. Cipolla MJ, Bishop N, Chan SL. Effect of pregnancy on autoregulation of cerebral blood flow in anterior versus posterior cerebrum. Hypertension 2012;60:705-11

30. Porcello Marrone LC, Gadonski G, de Oliveira Laguna G, et al. Bloodbrain barrier breakdown in reduced uterine perfusion pressure: a possible model of posterior reversible encephalopathy syndrome. J Stroke Cerebrovasc Dis 2014;23:2075-79

31. Ishikura K, Ikeda M, Hamasaki Y, et al. Posterior reversible encephalopathy syndrome in children: its high prevalence and more extensive imaging findings. Am J Kidney Dis 2006;48:231-38

32. McKinney AM, Kieffer SA, Paylor RT, et al. Acute toxic leukoencephalopathy: potential for reversibility clinically and on MRI with diffusion-weighted and FLAIR imaging. AJR Am J Roentgenol 2009;193:192-206

33. Alhilali LM, Reynolds AR, Fakhran S. A multi-disciplinary model of risk factors for fatal outcome in posterior reversible encephalopathy syndrome. J Neurol Sci 2014;347:59-65

34. Gao B, Liu FL, Zhao B. Association of degree and type of edema in posterior reversible encephalopathy syndrome with serum lactate dehydrogenase level: initial experience. Eur J Radiol 2012;81: 2844-47

35. Lv C, Gao B. Serum lactate dehydrogenase as a predictor of outcome in posterior reversible encephalopathy syndrome: imperative to unify. AJNR Am J Neuroradiol 2015;36:E29-30 